American J. of Engineering and Applied Sciences 3 (1): 98-101, 2010

ISSN 1941-7020

(C) 2010 Science Publications

\title{
Single Beam Z-Scan Measurements of Nonlinear Refraction and Nonlinear Absorption Coefficients in Silver Nano-Fluid
}

\author{
Esmaeil Shahriari and W. Mahmood Mat Yunus \\ Department of Physics, Faculty of Science, \\ University Putra Malaysia, 43400 UPM, Serdang, Malaysia
}

\begin{abstract}
Problem statement: The nonlinear refractive index and nonlinear absorption are two important optical phenomena which are extensively used in optical switching, optical limiting and labeling. Approach: In the present research we had measured nonlinearity of Ag nano-fluid prepared using $\gamma$-irradiation method. The measurement was carried out using a single beam z-scan technique. Under a CW laser beam excitation operated at $532 \mathrm{~nm}$ with the power output of $40 \mathrm{~mW}$, a closed aperture setup was used for thermal-induced nonlinear refractive index and an open aperture setup was applied for measuring nonlinearity absorption. Results: We measured the nonlinear refraction coefficient and nonlinear absorption coefficients for silver nano-fluid at concentration $6.475 \times 10^{-3} \mathrm{M}$, the values obtained were $-6.173 \times 10^{-8} \mathrm{~cm}^{2} \mathrm{~W}^{-1}$ and $7.994 \times 10^{-3} \mathrm{~cm} \mathrm{~W}^{-1}$ respectively. Conclusion: The nonlinear refraction coefficient with the negative sign indicates the self-defocusing phenomenon. The experimental data from open aperture measurement showed that a two photon absorption phenomenon. These results showed that the Ag nano-fluid has significant values of nonlinear refractive index and nonlinear absorption, thus it could be good candidate for optical devices.
\end{abstract}

Key words: Self-defocusing, nonlinear optics, nano-metal, thermal effects

\section{INTRODUCTION}

The single beam Z-scan technique was developed by the Sheik-Bahae et al. (1989). This method is a simple and effective tool for determining the nonlinear properties. It has been used widely in material characterization because it provides not only the magnitudes of the real and imaginary parts of the nonlinear susceptibility, but also the sign of the real part (Sutherland et al., 2003; Li et al., 1994; Marder and Torruellas, 1997; He et al., 1997). This method, utilizes a tightly focused laser beam that is intense enough to access nonlinearities in a sample. As the sample passes through the focal point of the beam, changes in its transmittance due to Nonlinear Absorption (NLA) and Nonlinear Refraction (NLR) are measured by an open aperture and closed aperture, respectively. In the open aperture technique, after the beam is passed through the sample, it is focused directly into a detector. As the sample travels through the focus of the initial beam, the transmittance either increases or decreases (depending on the nonlinearity of the sample) and the detector receives more or less light than the linear transmittance, yielding a hump or dip in the curve of transmittance as a function of sample position. For NLR, after the beam passing through the sample, it is attenuated by a semiclosed aperture that usually allows about $30 \%$ of the initial beam to be detected by the detector. With this, due to converging and diverging of the beam (allowing more and less of the beam to pass through the aperture, respectively) made changes in the refractive index. A pre-focal valley and post-focal peak is observed for a positive change in refraction and a pre-focal peak and a post-focal valley is observed for a negative change in refraction.

There has been a large necessity for nonlinear optical materials that can be used with low intensity lasers for applications such as phase conjugation, image processing and optical switching (Kramer et al., 1986; Hernandez et al., 1998; Yin et al., 2000 Ryasnyansky and Palpant, 2005). Metal nano-fluids has demonstrated a vast range of applications such as, the labeling of biological molecules, surface enhanced Raman scattering, optical limiter and optical photonics devices (Kovsh and Yang, 1999; Kovsh and Yang, 1999). In this study, we report the nonlinear refractive index and nonlinear absorption coefficient of $\mathrm{Ag}$ nano-fluid prepared using $\gamma\left({ }^{60} \mathrm{Co}\right.$-rays $)$ radiation at $50 \mathrm{kGy}$ level.

Corresponding Author: Esmaeil Shahriari, Department of Physics, Faculty of Science, University Putra Malaysia, 43400 UPM, Serdang, Malaysia Tel : +(603)89466684 


\section{MATERIALS AND METHOD}

To prepare Ag nano-fluid sample, $55 \mathrm{mg}$ of silver nitrate $\left(\mathrm{AgNO}_{3} \quad\right.$ Aldrish-99\%), $4 \mathrm{~g}$ of polyvinylpyrrolidone (PVP, MW 29,000 Aldrish) and $1 \mathrm{~mL}$ isopropanol were used. The PVP and isopropanol were used as a colloidal stabilizer and radical scavenger of hydroxyl radical respectively. The PVP solution was made by dissolving PVP powder in $50 \mathrm{ml}$ of deionized water at room temperature. The solution was stirred for $2 \mathrm{~h}$ and was bubbled with nitrogen gas $(99.5 \%)$ for removing the oxygen gas. The $\gamma$-radiation $\left({ }^{60} \mathrm{Co}\right.$-rays) source is an effective tool for polymerization process and reducing agent. Silver nitrate $(\mathrm{AgNO} 3)$ was added into PVP solution and the concentration of $\mathrm{Ag}$ nanoparticles in solution was calculated to be $6.475 \times 10^{-3}$ M. Sample was then irradiated with $\gamma$ radiation at a dose of $50 \mathrm{kGy}$. In this process, $\gamma$ irradiation produces hydrated electrons that reduce the silver ions to silver atoms, which then aggregated in the solution. The average diameter of $\mathrm{Ag}$ nanoparticles was measured using Nanophox Machine (Sympatec GmbH, D-38678) and the particle average size was recorded as $42.3 \mathrm{~nm}$. The linear absorption spectra Ag nano-fluid sample was measured by conventional method on the basis of $\alpha=-(1 / \mathrm{L}) \operatorname{Ln}\left(\mathrm{I}_{\mathrm{o}} / \mathrm{I}\right)$ in the linear regime of the experiment.

Figure 1 shows the experimental set up of a single beam Z-scan experiment used in the present measurement. The experiment was carried out at room temperature using a $\mathrm{CW}$ beam diode laser operated at $532 \mathrm{~nm}$ wavelength (Coherent Compass SDL-532-150T). The beam was focused to a small spot using a lens and the sample was moved along the $\mathrm{z}$-axis by a motorized translational stage. At the focus point the power output of the laser beam was measured to be $40 \mathrm{~mW}$.

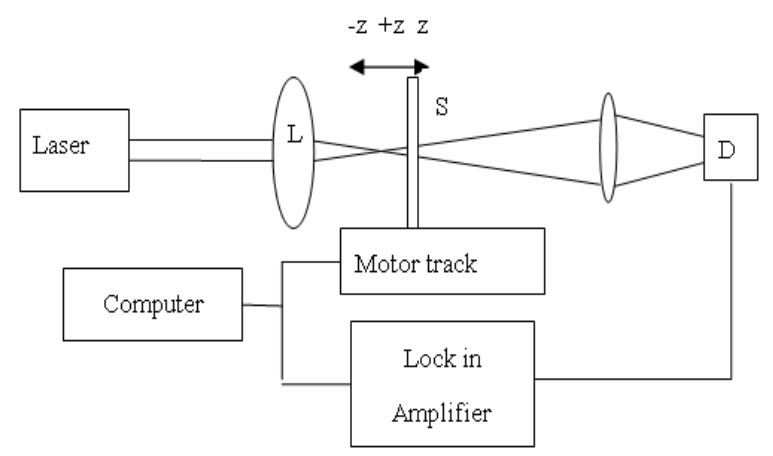

Fig. 1: Schematic diagram of a single beam Z-scan experiment setup: L: Lens; S: Sample; A: Aperture; D: Detector
The transmitted light in the far field passed through the aperture and the beam intensity was recorded by a photodiode detector, $\mathrm{D}$. The laser beam waist $\omega_{0}$ at the focus length was measured to be $24.4 \mu \mathrm{m}$ and the Rayleigh length was found to satisfy the basic criteria of a z-scan experiment. A quartz optical cell containing specimen solution was translated across the focal region along the $\mathrm{z}$-axis direction.

\section{RESULTS}

Figure 2 shows the open aperture z-scan experimental data obtained for $\mathrm{Ag}$ nano-fluid with concentration of $6.475 \times 10^{-3} \mathrm{M}$ irradiated at $50 \mathrm{kGy}$. The nonlinear absorption coefficient $\beta$ can be obtained from this open aperture Z-scan data by fitting the normalized transmittance data to the open aperture formula given as (Sheik-Bahae et al., 1989; SheikBahae et al., 1990):

$\mathrm{T}(\mathrm{z}, \mathrm{s}=1)=\sum_{\mathrm{m}=0}^{\infty} \sum_{\mathrm{m}=0}^{\infty} \frac{\left[-\mathrm{q}_{0}(\mathrm{z})\right]^{\mathrm{m}}}{(\mathrm{m}+1)^{3 / 2}}\left|\mathrm{q}_{0}(\mathrm{z})\right|<1$

Where:

$\mathrm{q}_{0}(\mathrm{z})=\mathrm{I}_{0} \beta \mathrm{L}_{\text {eff }} /\left(1+\mathrm{z}^{2} / \mathrm{z}_{0}^{2}\right)$

$\mathrm{z}_{0} \quad=\mathrm{k} \omega_{0}^{2} / 2$ is the diffraction length of the beam

$\mathrm{k}=2 \pi / \lambda$ is the wave factor

$\omega_{0}=$ The beam waist radius at the focal point

$L_{\text {eff }}=(1-\exp (-\alpha \mathrm{L})) / \alpha$ is the effective thickness of the sample

The solid line in Fig. 2 is theoretical curve while the symbol is the experimental data. The nonlinear absorption coefficient obtained was $7.994 \times 10^{-3} \mathrm{~cm} \mathrm{~W}^{-1}$.

The nonlinear refractive index can be calculated from the normalized transmittance data of closed aperture measurement which can be written as follows (Sheik-Bahae et al., 1990):

$$
\mathrm{T}(\mathrm{z}, \Delta \varphi)=1-\frac{4 \Delta \varphi_{0} \mathrm{x}}{\left(\mathrm{x}^{2}+1\right)\left(\mathrm{x}^{2}+9\right)}
$$

where, $\Delta \varphi_{0}$ is the on axis phase change at the focus point. This produces a symmetry $\Delta \mathrm{T}_{\mathrm{p}-\mathrm{v}}$ (peak-valley) curve where the nonlinear refractive index, $\mathrm{n}_{2}$ can be calculated using simple expression reported by SheikBahae et al. (1989); Sheik-Bahae et al. (1990) that is:

$$
\mathrm{n}_{2}=\frac{\Delta \mathrm{T}_{\mathrm{p}-\mathrm{v}}}{0.406(1-\mathrm{s})^{0.25} \mathrm{~kL}_{\text {eff }} \mathrm{I}_{\mathrm{o}}}
$$

where, $\mathrm{s}$ is the aperture in linear transmittance regime. 


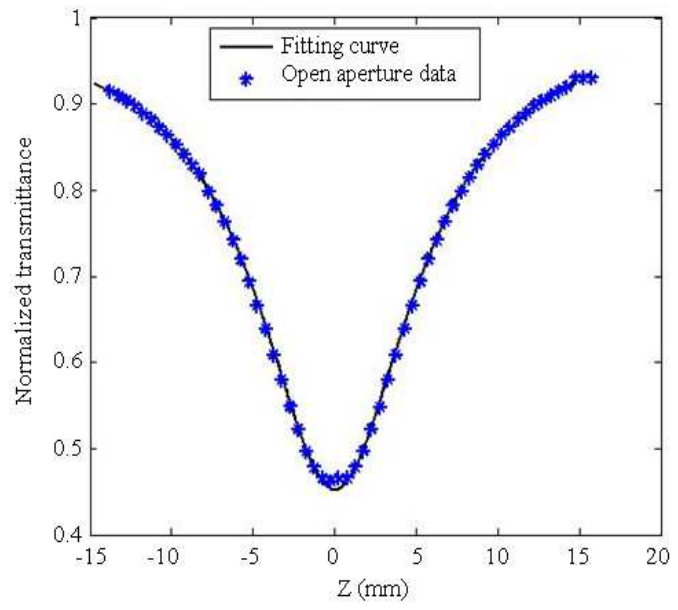

Fig. 2: Open aperture Z-scan curve for Ag nanoparticle measured at a concentration of $6.475 \times 10^{-3} \mathrm{M}$ irradiated at $50 \mathrm{kGy}$. Solid line is the fitted curve using Eq. 1

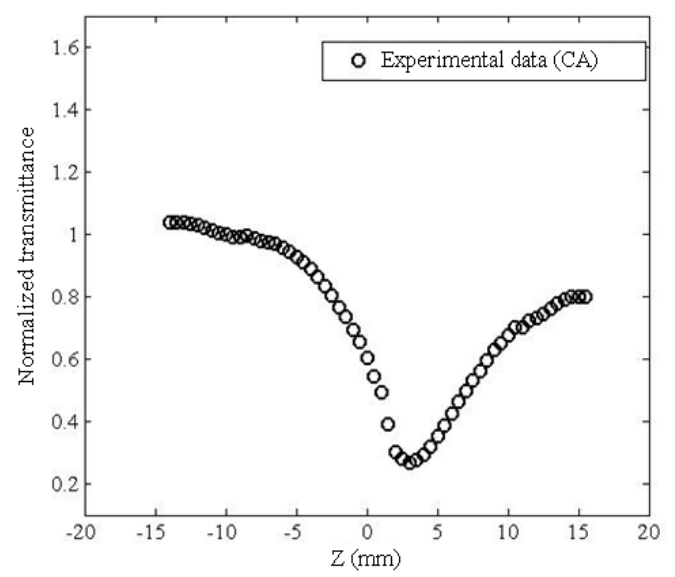

Fig. 3: Closed aperture experimental data of Ag nanofluid measured at concentration of $6.475 \times 10^{-3}$ M. The average particle size is $42.3 \mathrm{~nm}$

Figure 3 shows the transmittance curves of a closed aperture z-scan obtained for $\mathrm{Ag}$ nano-fluid. The laser intensity measured was $I_{0}=4.27 \times 103 \mathrm{~W} \mathrm{~cm}^{-2}$ and the linear aperture transmittance was 0.10 . The curve is obviously asymmetry where there is a large suppressed in the transmittance peak and enhanced in the valley.

\section{DISCUSSION}

This behavior was due to the high nonlinear absorption phenomenon of the sample and can be solved by dividing the closed aperture (CA) experimental data of Fig. 3 over the open aperture (OA) experimental data of Fig. 2.

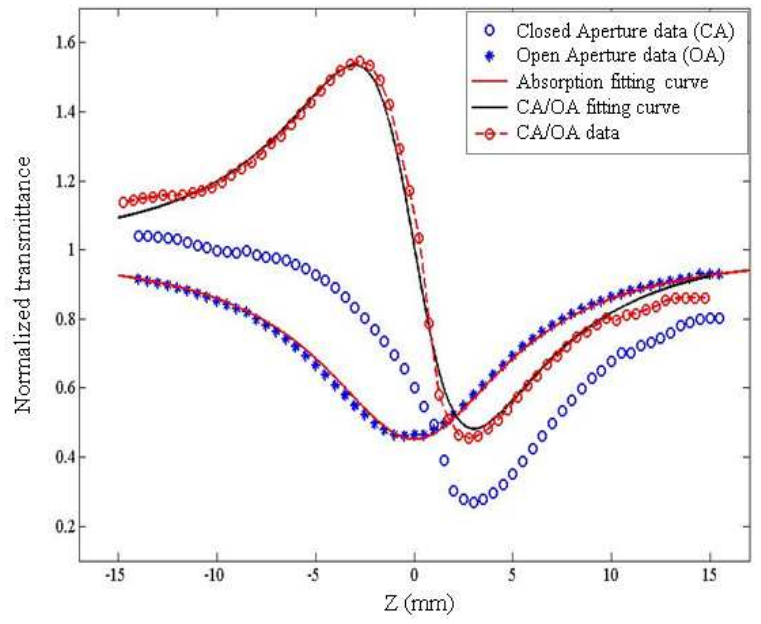

Fig. 4: Closed aperture Z-scan experimental data for $\mathrm{Ag}$ nano-fluid measured at concentration $6.475 \times 10^{-3} \mathrm{M}$. The solid line is the theoretical curve calculated using Eq. 1 and 2 respectively

Figure 4 shows a perfect symmetry experimental curve was produced by dividing the closed aperture to open aperture experimental data of Fig. 3 and 2. By measuring the $\Delta \mathrm{T}_{\mathrm{p}-\mathrm{v}}$ from CA/OA fitted curve in Fig. 4 we obtained the third order nonlinear refractive index, $\mathrm{n}_{2}$ as $-6.173 \times 10^{-8} \mathrm{~cm}^{2} \mathrm{~W}^{-1}$. The negative sign confirms the nonlinear phenomenon was due to the selfdefocusing process.

This result shows that the Ag nano-fluid with the particle size of $42.3 \mathrm{~nm}$ gives significant values of nonlinear refractive index and nonlinear absorption. Thus sample preparation technique will be an important aspect in studying nonlinear optical properties of metal nano-particle solution. Since the $\gamma$ - radiation technique can easily control the particle size of metal nanoparticle in solution, a specific particle size can be produced for specific nonlinear optical devices. This will offer a further nonlinearity study in metal nanofluids for specific applications.

\section{CONCLUSION}

The third-order nonlinear refraction and twophoton absorption coefficient were measured for the $\mathrm{Ag}$ nano-fluid using a single bean Z-scan technique. The values of nonlinear refractive index, $\mathrm{n}_{2}$ and nonlinear absorption coefficient for $\mathrm{Ag}$ nano-fluid measured at $532 \mathrm{~nm}$ laser excitation were $-6.173 \times 10^{-8} \mathrm{~cm}^{2} \mathrm{~W}^{-1}$ and $7.994 \times 10^{-3} \mathrm{~cm} \mathrm{~W}^{-1}$ respectively. The experiment also confirmed that the nonlinear phenomenon was caused by self-defocusing process. These results show that the 
$\mathrm{Ag}$ nano-fluid with a particle size of $42.3 \mathrm{~nm}$ is a promising material for optical devices.

\section{ACKNOWLEDGEMENT}

We gratefully acknowledge the Department of Physics, UPM for providing the research facilities to enable us to carry out this research. One of the authors (W. M. Mat Yunus) would like to acknowledge the MOSTI for the financial support through Fundamental research grant (01-11-08-664FR/5523664).

\section{REFERENCES}

He, G.S., L.X. Yuan, Y.P. Cui and P.N. Prasad, 1997. Studies two-photon pumped frequency unconverted. Applied Phys., 81: 2529-2537. DOI: 10.1063/1.364303

Hernandez, F.E., A.O. Marcano, Y. Alvarado, A. Biondi and H. Maillotte, 1998. Measurment of nonlinear refraction index and two-photon absorption in a novel organometallic compound. Opt. Commun., 152: 77-82.

Hernandez, F. and A.O. Marcano, 1998. Measurement of nonlinear refraction index and two-photon absorption in a novel organometallic compound. Opt. Commun., 152: 77-82.

Kramer, M.A., W.R. Tompkin and R.W. Boyd, 1986. Nonliner optical interactions in fluorescein-doped boric acid glass. Phys. Rev. A., 34: 2026-2031. DOI: 10.1103/PhysRevA.34.2026

Kovsh, D. and S. Yang, 1999. Nonlinear optical beam propagation for optical limiting. Applied Opt., 38: 5168-5180. DOI: 10.1364/AO.38.005168

Li, C. L. Zhang, M. Yang, H. Wang and Y. Wang, 1994. Dynamic and steady-state behavior of reverse saturable absorption in metallophthalocyanine. Phys. Rev. A., 49: 1149-1157. DOI: 10.1103/PhysRevA.49.1149
Marder, S. and W. Torruellas, 1997. Large molecular third-order optical nonlinearities in polarized carotenoids. Science, 276: 1233-1236. DOI: 10.1126/science. 276.5316 .1233

Ryasnyansky, A.I. and B. Palpant, 2005. Theoretical investigation of the off-axis $\mathrm{z}$-scan technique for nonlinear optical refraction measurement. Applied Opt., 45: 2773-2776. DOI: 10.1364/AO.45.002773

Sheik-Bahae, M., A.A. Said, E.W. Van Stryland, 1989. High sensitivity single beam $\mathrm{n}_{2}$ measurements. Opt. Lett., 14: 955-957. DOI: 10.1364/OL.14.000955

Sheik-Bahae, M., A.A. Said, T. Wei, D.J. Hagan and E.W. van Stryland, 1990. Sensitive measurement of optical nonlinearities using a single beam. IEEE J. Quant. Elect., 26: 760-769. DOI: 10.1109/3.53394

Sutherland, R.L. and D. McLean et al., 2003. Handbook of Nonlinear Optics. 2nd Edn., CRC Press, ISBN: 0-8247-4243-5, pp: 457.

Yin, M., H.P. Li, S.H. Tang and W. Ji, 2000. Determination of nonlinear absorption and refraction by single Z-scan method. Applied Phys. B., 70: 587-591. DOI: 10.1007/s003400050866 